\title{
Affine-Goldstone/quartet-metric gravity and beyond
}

\author{
Yury F. Pirogov \\ Theory Division, Institute for High Energy Physics of NRC Kurchatov Institute, Protvino, Russia
}

\begin{abstract}
As a group-theoretic foundation of gravity, it is considered an affine-Goldstone nonlinear model based upon the nonlinear realization of the global affine symmetry spontaneously broken at the Planck scale to the Poincare symmetry. It is shown that below this scale the model justifies and elaborates an earlier introduced effective field theory of the quartet-metric gravity incorporating the gravitational dark substances emerging in addition to the tensor graviton. The prospects for subsequent going beyond the nonlinear model above the Planck scale are indicated.
\end{abstract}

PACS: 04.50.Kd Modified theories of gravity, 95.35.+d Dark matter, 95.36.+x Dark energy.

\section{Introduction: GR and beyond}

General Relativity (GR) is the well-stated contemporary basis of gravity remaining up-todate in a position to successfully cope with the bulk of the astrophysical and cosmological manifestations of gravity. Nevertheless, it may be argued that an underlying nature of gravity beyond GR is still obscure. The reason may come from the impressive recent achievements in the observational astrophysics and cosmology. Namely, the advent in the Universe of the elusive dark substances, such as dark matter (DM) and dark energy (DE), in an amount disproportionately large ( $\sim 95 \%$ of the total energy) compared to the ordinary matter $(4 \div 5 \%)]^{1}$ with their nature remaining moreover completely obscured, causes some (still mainly theoretical) tension within GR. The predominant abundance of such the ad hock dark substances, though quite legitimate in the GR framework, may be a hint from the Nature at a necessity of going beyond GR, with the elusive dark substances being nothing but an integral part of the modified gravity itself.

With such an aim in mind, as a modification of the (metric) GR there was recently proposed in refs. [2, 3] the effective field theory (EFT) of the spontaneously broken quartetmodified GR (or, in short, the quartet-metric gravity). The latter is based originally upon the three physical concepts. First, there exist in spacetime the distinct dynamical coordinates given by a scalar quartet (in the four spacetime dimensions). Such coordinates are ultimately associated with the vacuum treated as the dynamical one on par with the other fields. Second, the diffeomorphism symmetry of the quartet-modified GR undergoes the spontaneous symmetry breaking (SSB), with the scalar quartet playing

\footnotetext{
${ }^{1}$ See, e.g., [1].
} 
the role of the Higgs-like fields for gravity. Third, the physical gravity components arising from the gauge ones of metric due to SSB serve as the gravitational dark substance of the Universe. It was argued that such a constructed EFT of gravity may give rise to a large variety of manifestations beyond GR, to consistently study which presents a challenge. At that, the latter is hampered by the fact, that by the very construction the conventional EFT frameworks inevitably contain a number of the ad hock assumptions and parameters implying, conceivably, a necessity of further elaborating such a theory, as well as its foundations.

In the latter respect note, that GR (like its direct siblings) is well-known to be based upon the pseudo-Riemannian/metric paradigm of gravity, which is conventionally assumed to be an attribute of gravity. On the other hand, already long ago there was put forward [4, 5] an alternative, group-theoretic/Nambu-Goldstone (NG) paradigm, wherein gravity has an NG origin being based upon the spontaneous breaking at the Planck scale of the global affine symmetry to the Poincare one, with the emergent NG boson associated with graviton. The latter paradigm proves to be nicely fitted to gravity, naturally incorporating the generic signatures of GR: the symmetric second-rank tensor field possessing the self-interactions through a derivative in the ratio to the Planck mass treated as a scale of the affine SSB. By means of the group-theoretic techniques of the nonlinear realizations (NRs) and nonlinear models (NMs) for SSB [6]-9] this allows to justify GR as the metric theory of gravity, with the graviton being ultimately a Goldstone field. The question then arises: to what extent such an alternative approach to gravity may be of use for going beyond GR, as was advocated above.2 ${ }^{23}$

To this end, in the present paper we merge the two above-mentioned routes of the GR modification, so to say, "in width" and "in depth". While the quartet-modified GR (like GR itself and its direct siblings) is originally based upon the pseudo-Riemannian/metric paradigm in the conventional EFT frameworks, we are now going to consider a more advanced EFT of gravity (encompassing the plain one) based upon the group-theoretic/NG paradigm in the NM frameworks. At that, due to the internal and external symmetries in the case at hand coinciding we construct such an NM for consistency in the two steps. In Sec. 2, in an auxiliary affine space, a progenitor of the Lorentz spacetime, we start by presenting NRs for the spontaneously broken global affine symmetry and then build an affine-Goldstone NM. In Sec. 3, such a construction is proliferated to an arbitrary world manifold, a progenitor of the pseudo-Riemannian spacetime. It is shown that NM so constructed supersedes the earlier introduced EFT of the quartet-metric gravity, with a clarification and elaboration of the latter. For illustration, the two extreme cases of the quartet-metric gravity are exposed in more detail. In Summary, there are collected the main advantages of presenting the quartet-metric gravity as NM in the group-theoretic/NG paradigm vs. EFT in the pseudo-Riemannian/metric paradigm. Finally, we point out towards a conceivable prospects of going beyond the NM frameworks above the Planck scale towards an underlying theory for the emergent gravity and spacetime. All the constructions for the affine-Goldstone NM are presented throughout in a nutshell directly adopted to the case at hand. For completeness, in Appendix there are exposed in a more generality the group-theoretic basics concerning SSB, NRs and NMs.

\footnotetext{
${ }^{2}$ For gravity as an affine-Goldstone phenomenon and beyond, cf., 10 .

${ }^{3}$ For graviton as a Goldstone field in the Lorentz gauge theory, cf., [11.
} 


\section{Affine-Goldstone NM}

\subsection{Affine-to-Poincare SSB}

Let us first indicate the basic results in the affine space for NM to be used subsequently in constructing the modified gravity in the world manifold. $\left.\right|^{4}$ To start, let $R^{4}=\left\{z^{a}\right\}$, be an auxiliary affine space. $5^{5}$ with the coordinates $z^{a}$ marked by the indices $a, b, \ldots=0, \ldots, 3$, acted upon by the global affine transformations $(A, C) \in \operatorname{IGL}(4, R) \equiv \operatorname{Aff}\left(R^{4}\right)$ as $z \rightarrow A z+C$, or in the full notation

$$
(A, C): z^{a} \rightarrow A^{a}{ }_{b} z^{b}+C^{a} .
$$

At that, the index 0 is a priori nothing but a notation. The NM under construction is based upon the SSB pattern ${ }^{6}$

$$
G \equiv G L(4, R) \rightarrow H \equiv S O(1,3)
$$

with $S O(1,3) \subset G L(4, R)$ the residual (global) Lorentz subgroup. Remaining unbroken, the latter serves as a classification group for the field representations. Just after a particular embedding of the unbroken subgroup $S O(1,3)$ into the broken $G L(4, R)$ the affine space gets converted into the Lorentz spacetime, with a foliation onto the time and space: $z^{a}=\left(z^{0}, z^{i}\right), i=1,2,3$, where the affine index 0 is assumed to be chosen so as to acquire its conventional time meaning. The respective NM $G / H=G L(4, R) / S O(1,3)$ describes the SSB $G \rightarrow H$, with the appearance of the $d_{G / H}=d_{G}-d_{H}=10$ NG bosons associated ultimately with the (tensor) graviton and the additional gravitational dark components..$^{7}$

\subsection{Quasi-symmetric NR}

The construction of the proper NM, call it the affine-Goldstone one, follows (with slight modifications) to the general procedure of NRs (see, e.g., Appendix). What is of specifics

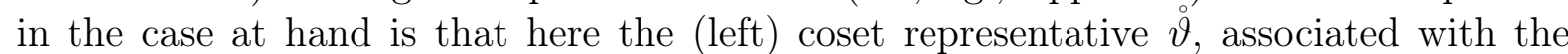
NG field, may be parametrized as a $4 \times 4$ (local) matrix $\mathfrak{\vartheta}_{(b)}^{a}(z)$ transforming under $A \in G L(4, R)$ as $\grave{\vartheta} \rightarrow A \grave{\vartheta} \Lambda^{-1}(A, \grave{\vartheta})$. or in the full notation

$$
A: \stackrel{\circ}{\vartheta}_{(b)}^{a}(z) \rightarrow \stackrel{\circ}{(b)}_{(b)}^{a}(A z)=A_{c}^{a}{ }_{(d)}^{c}(z) \Lambda_{b}^{-1 d}(A, \stackrel{\circ}{\vartheta}(z)),
$$

with both the field $\grave{\vartheta}$ and the coordinate $z$ transforming under $A$. At that, $\Lambda=\Lambda(A, \grave{\vartheta}) \in$ $S O(1,3)$ is to be properly defined (henceforth, the term nonlinear realization) to specify a particular NR. In what is shown above and what follows, an affine index in the parentheses indicates that it undergoes transformations just under the residual Lorentz subgroup $S O(1,3)$. Only such indices are allowed to be raised and lowered by means of the Lorentzinvariant Minkowski symbol $\eta^{a b}$ (and, respectively, $\eta_{a b}$ ) without an explicit violation

\footnotetext{
${ }^{4}$ Taking these results for granted one may directly consider their application in Sec. 3 .

${ }^{5}$ Such an affine realization space may physically be thought of as corresponding to a putative primary metricless world (or a "bare" vacuum).

${ }^{6}$ In fact, we consider the full affine and inhomogeneous Lorentz (Poincare) groups. But remaining unbroken, the inhomogeneous parts of the groups are explicitly omitted.

${ }^{7}$ Note that all the consideration is technically insensitive to the dimension of spacetime $d \geq 3$, as well as to its signature $(p, q), p+q=d$. For this reason, the quartet-metric gravity, containing in metric the gravity components in excess of the tensor graviton, may rather be called the multi-component gravity.
} 
of the affine symmetry. With $\stackrel{\circ}{(b)}^{a}$ representing a group element it has an inverse $\grave{\vartheta}_{b}^{(a)}$ transforming under $A \in G L(4, R)$, in short, a $a^{8} \dot{\vartheta}^{-1} \rightarrow \Lambda(A, \grave{\vartheta}) \dot{\vartheta}^{-1} A^{-1}$.

An arbitrary $4 \times 4$ matrix contains a priori sixteen components. To fix a (left) coset representative $\vartheta$ there should be leaved in the matrix just ten independent components, equal to the difference of the dimensions of the linear and Lorentz groups, through imposing six auxiliary conditions. In the case at hand the most natural choice is the quasi-symmetric condition, in short, $\stackrel{\vartheta}{\eta}_{\eta}=(\stackrel{\vartheta}{\eta})^{T}=\eta \vartheta^{T}$, or

$$
\stackrel{\vartheta}{\vartheta}_{(c)}^{a} \eta^{c b}=\stackrel{\circ}{\vartheta}_{(c)}^{b} \eta^{c a}
$$

eliminating the (quasi)-anti-symmetric part present in the arbitrary $4 \times 4$ matrix. With account for the pseudo-orthogonality property of $\Lambda \in S O(1,3)$, in short, $\eta \Lambda^{T} \eta=\Lambda^{-1}$, or

$$
\eta^{a c} \Lambda_{c}^{d} \eta_{d b}=\Lambda_{b}^{-1 a}
$$

to preserve the imposed condition under an arbitrary $A$ there should fulfill

$$
A \grave{\vartheta} \eta \Lambda^{T}=\Lambda \grave{\vartheta} \eta A^{T} .
$$

Under restriction by $A=\Lambda_{0}$, with an arbitrary global $\Lambda_{0} \in S O(1,3)$, we clearly get $\Lambda=\Lambda_{0}$ implying, in particular, $\Lambda=I$ at $A=I$, with $\grave{\vartheta}$ remaining unchanged. A general solution to eq. (6) may be looked for by perturbations, uniquely at least in a vicinity of unity. The resulting $\Lambda=\Lambda(A, \vartheta)$ defines the particular NR, which reduces to the linear representation when restricted by the unbroken Lorentz subgroup;

$$
\Lambda_{0}: \stackrel{\wp}{\rightarrow} \Lambda_{0} \grave{\vartheta} \Lambda_{0}^{-1},
$$

or, with account for the pseudo-orthogonality of $\Lambda_{0}$, as the symmetric Lorentz tensor:

$$
\Lambda_{0}: \stackrel{\vartheta}{\eta} \rightarrow \Lambda_{0} \grave{\vartheta} \eta \Lambda_{0}^{T}
$$

At last, decomposing $\grave{\vartheta}$ in the weak-field limit as

$$
{\stackrel{\circ}{\vartheta_{(c)}^{a}}}^{c b} \simeq \eta^{a b}+\chi^{a b}
$$

with the symmetry $\chi^{a b}=\chi^{b a}$ being preserved by the classification Lorentz subgroup, we may interpret $\chi$ as a Lorentz-tensor NG boson for SSB at hand.

Imposing other auxiliary conditions we may similarly get other particular NRs, all of them being equivalent (within the full NM). Dealing exclusively with a particular NR may be cumbersome. For this reason, we get rid of the explicit auxiliary conditions and consider below (in accord with the general theory of NRs) a linearized realization explicitly equivalent to any particular NR, but being, in fact, much simpler.

\subsection{Local-Lorentz linearization}

Instead of a (left) coset representative $\grave{\vartheta}(z)$, let us now choose as a field variable for SSB $G \rightarrow H$ the (left) coset itself, i.e., the whole subset (not, generally, a subgroup) of the elements of $G$ equivalent modulo the (right) multiplication by $H$ and containing $\dot{\vartheta}$ as a representative: $\vartheta \equiv\left\{\grave{\vartheta} \Lambda^{-1}\right\}, \Lambda \in H=S O(1,3)$. To this end, define for the case at hand

\footnotetext{
${ }^{8}$ We designate the inverse matrix $\grave{\vartheta}_{b}^{(a)}$ in a short-hand notation as $\grave{\vartheta}^{-1}$, etc.
} 
as a new field variable the $4 \times 4$ local matrix $\vartheta_{\alpha}^{a}(z)$ (with an inverse $\vartheta_{a}^{\alpha}$ ) transforming under $A \in G L(4, R)$ up to an arbitrary $\Lambda(z) \in S O(1,3)_{\text {loc }}$, in short, as $\vartheta \rightarrow A \vartheta \Lambda^{-1}(z)$, or

$$
(A, \Lambda(z)): \vartheta_{\alpha}^{a}(z) \rightarrow \vartheta_{\alpha}^{\prime a}(A z)=A_{b}^{a} \vartheta_{\beta}^{b}(z) \Lambda^{-1 \beta}{ }_{\alpha}(z),
$$

(respectively, $\vartheta^{-1} \rightarrow \Lambda(z) \vartheta^{-1} A^{-1}$ ). In what is shown above, $S O(1,3)_{\text {loc }}$ is an auxiliary local Lorentz group (not a subgroup of $G L(4, R)$ as before). At that, though $\vartheta_{\alpha}^{a}(z)$ contains formally sixteen components, six of them can be eliminated by means of the auxiliary transformations $\Lambda(z) \in S O(1,3)_{\text {loc }}$ leaving as required precisely ten independent components. The remaining components may ultimately be associated with the NG boson arising under SSB $G L(4, R) \rightarrow S O(1,3)$. Namely, fixing properly a gauge for $S O(1,3)_{\text {loc }}$ we can get any particular NR. Thus, transforming $\vartheta \rightarrow \vartheta \Lambda^{-1}$, with $\Lambda \in$ $S O(1,3)_{\text {loc }}$ satisfying, with account for the pseudo-symmetricity of $\AA$, to the relation, in short, $\vartheta \eta \AA^{T}=\left(\vartheta \eta \AA^{T}\right)^{T}=\grave{\Lambda} \eta \vartheta^{T}$, or

$$
\left.\vartheta_{\alpha}^{a} \eta^{\alpha \gamma} \stackrel{\Lambda}{\Lambda}_{\gamma}^{\beta}\right|_{\beta=b}=\left.\vartheta_{\beta}^{b} \eta^{\beta \gamma} \stackrel{\Lambda}{\alpha}_{\gamma}^{\alpha}\right|_{\alpha=a},
$$

we can achieve that $\grave{\vartheta} \equiv \vartheta \AA^{-1}$ gets quasi-symmetric, ${ }_{\eta} \eta=(\grave{\vartheta} \eta)^{T}=\eta \vartheta^{T}$, recovering thus the quasi-symmetric NR. In the same vein, we can get any other particular NR, all of them (in accord with the general NR theory) being equivalent to each other and to the local-Lorentz linearized realization.

In what follows, we will use such a realization, with the symmetry group in the factorized affine-Lorentz form: 9

$$
G \times H_{\mathrm{loc}}=G L(4, R) \times S O(1,3)_{\mathrm{loc}} .
$$

The SSB pattern eq. (2) and the hidden local symmetry in eq. 12) may be treated as an essence of gravity, succinctly encompassing the bulk of the gravity appearance, modulo the world environment (see, Sec 3). According to eq. (12), there can be envisaged the three generic types of the (finite dimensional) affine-Lorentz fields: the affine protometric, the affine tensors and Lorentz spinors, to be specified below.

\subsection{Affine-Lorentz fields}

\subsubsection{Affine protometric}

To describe the dynamics of the affine NG field $\vartheta$ consider, in accord with the general prescription, the (slightly modified) so-called Maurer-Cartan form, in short, $\Omega_{a}=\vartheta^{-1} \partial_{a} \vartheta \eta$, or

$$
\Omega_{c}^{\alpha \beta}=\vartheta_{d}^{\alpha} \partial_{c} \vartheta_{\gamma}^{d} \eta^{\gamma \beta}
$$

According to eq. (10), $\Omega_{a}$ is a vector under $G L(4, R)$ and, with account for the pseudoorthogonality of $\Lambda$, transforms under $S O(1,3)_{\text {loc }}$ inhomogeneously as

$$
(A, \Lambda(z)): \Omega_{c}(z) \rightarrow \Omega_{c}^{\prime}(A z)=A_{c}^{-1 b}\left(\Lambda \Omega_{b} \Lambda^{T}+\Lambda \partial_{b} \eta \Lambda^{T}\right) .
$$

Decomposing $\Omega_{c}$ in the symmetric and anti-symmetric parts, in short, $\Omega_{c}^{ \pm} \equiv\left(\Omega_{c} \pm \Omega_{c}^{T}\right) / 2$, or

$$
\Omega_{c}^{ \pm \alpha \beta} \equiv \frac{1}{2}\left(\Omega_{c}^{\alpha \beta} \pm \Omega_{c}^{\beta \alpha}\right)
$$

\footnotetext{
${ }^{9}$ At that, such an auxiliary non-compact local-Lorentz group $S O(1,3)_{\text {loc }}$, in distinction with a putative gauge one, is not equipped with the physical gauge fields, so that in the case at hand there appears no apparent problems with unitarity.
} 
we can see from eq. (14) that the symmetric part transforms homogeneously under $\Lambda(z)$, while the anti-symmetric part transforms inhomogeneously. This allows to use these parts separately. Namely, the inhomogeneously transforming anti-symmetric part $\Omega_{c}^{-}$ may serve as a Lorentz connection in the case of the fermion matter fields (see, later on). On the other hand, the symmetric part $\Omega_{c}^{+}$being a Lorentz tensor may be used to describe the NG field $\vartheta$ by its own through constructing the arbitrary local-Lorentz invariant combinations.

Otherwise, due to a freedom (within the full NM) of choosing the field variables, to facilitate the procedure we may start directly from the local-Lorentz invariant combination in the short-hand notation $\theta=\vartheta \eta \vartheta^{T}, \theta=\theta^{T}$, or

$$
\theta^{a b} \equiv \vartheta_{\alpha}^{a} \eta^{\alpha \beta} \vartheta_{\beta}^{b}, \quad \theta^{a b}=\theta^{b a}
$$

with the inverse $\theta^{-1}=\vartheta^{-1 T} \eta \vartheta^{-1}, \theta^{-1}=\theta^{-1 T}$, or ${ }^{10}$

$$
\theta_{a b} \equiv \vartheta_{a}^{\alpha} \eta_{\alpha \beta} \vartheta_{b}^{\beta}, \quad \theta_{a b}=\theta_{b a} .
$$

So, instead of the affine-Lorentz bi-vector $\vartheta_{\alpha}^{a}$, corresponding to the affine NG boson, let us consider as a new field variable the genuinely-affine tensor $\theta^{a b}$ (or its inverse $\theta_{a b}$ ) corresponding to the point-like correlated symmetric pair of the NG bosons. Being symmetric, $\theta=\theta^{T}$, this tensor automatically contains the required number, ten, of the independent components irrespective of the particular gauge for $\Lambda(z)$. Under $G L(4, R) \times S O(1,3)_{\text {loc }}$ the field $\theta^{a b}$ transforms, in short, as

$$
(A, \Lambda(z)): \theta(z) \rightarrow \theta^{\prime}(A z)=A \theta(z) A^{T},
$$

with the symmetry of $\theta$ automatically preserved. As a result, NR for such a field becomes the conventional linear representation exclusively of the affine group. In the weak-field limit we have

$$
\theta^{a b} \simeq \eta^{a b}+2 \chi^{a b}
$$

with the NG boson $\chi^{a b}$ from eq. (9). Further, instead of a derivative of $\theta^{a b}$ we can equivalently use a genuinely-affine tensor $\Gamma_{a b}^{c}$ defined as

$$
\Gamma_{a b}^{c} \equiv \frac{1}{2} \theta^{c d}\left(\partial_{a} \theta_{b d}+\partial_{b} \theta_{a d}-\partial_{d} \theta_{a b}\right)
$$

so that inversely

$$
\partial_{c} \theta_{a b}=\theta_{a d} \Gamma_{b c}^{d}+\theta_{b d} \Gamma_{a c}^{d} .
$$

Looking at the first sight optional, the affine tensor $\Gamma_{a b}^{c}$ proves to be of a principle importance for the geometrical interpretation of the affine-Goldstone NM. The correlated pair of the NG fields, $\vartheta \eta \vartheta^{T}$ (resulting ultimately in the pseudo-Riemannian metric) may be called the affine protometric. Such a field being self-sufficient to construct in the affine space an NM by its own, proves at the same time to be inevitable to consistently incorporate other fields considered below.

\footnotetext{
${ }^{10}$ To distinguish from the affine-violating combination $\eta_{a c} \eta_{b d} \theta^{c d} \neq \theta_{a b}$, or in short $\eta \theta \eta \neq \theta^{-1}$.

${ }^{11}$ Clearly, the symmetric affine tensor $\Gamma_{a b}^{c}=\Gamma_{b a}^{c}$, like $\partial_{c} \theta_{a b}$, contains the same number, forty, of components as the symmetric affine-Lorentz tensor $\Omega_{a}^{+\alpha \beta}$, allowing ultimately to substitute each other.
} 


\subsubsection{Affine tensor fields}

Consider now an arbitrary affine-tensor field $\Phi_{b_{1}, \ldots}^{a_{1}, \ldots}(z)$ transforming under $A \in G L(4, R)$ independently in each of the upper and lower indices through $A$ and $A^{-1 T}$, respectively. The field $\theta^{a b}$ (or $\theta_{a b}$ ) may serve as a counterpart of the metric appearing due to the affine SSB in the originally metricless affine space. It allows to raise and lower the affine tensor indices to construct the bi-linear affine scalars, etc. E.g., by means of $\theta_{a b}$ we can construct from, say, an affine vector $U^{a}$ a quadratic combination $U^{a} U^{b} \theta_{a b}$ serving as an affine scalar, etc.

To facilitate the manipulations with such and similar constructions, in particular with their partial derivatives $\partial_{a}$, we can define in terms of the previously introduced affine tensor $\Gamma_{a b}^{c}(\theta)$ a counterpart of the covariant derivative. To this end, let us define the basic affine covariant derivative $\nabla_{a}$ for some vector fields $V_{a}$ and $U^{a}$ as follows:

$$
\begin{aligned}
\nabla_{a} U^{c} & =\partial_{a} U^{c}+\Gamma_{a d}^{c} U^{d}, \\
\nabla_{a} V_{b} & =\partial_{a} V_{b}-\Gamma_{a b}^{d} V_{d},
\end{aligned}
$$

so that $\nabla_{a}\left(U^{c} V_{c}\right)=\partial_{a}\left(U^{c} V_{c}\right)$, etc. Likewise, we can further proliferate the action of $\nabla_{a}$ on an arbitrary affine tensor $\Phi_{b_{1}, \ldots}^{c_{1}, \ldots}$ through the independent action on each of the indices as shown above. It follows hereof that

$$
\nabla_{a} \theta^{b c}=\nabla_{a} \theta_{b c}=0
$$

so that $\nabla_{a} V^{b} \equiv \nabla_{a} \theta^{b c} V_{c}=\theta^{b c} \nabla_{a} V_{c}$, etc. This allows, in particular, to disentangle the operations of differentiation and those of raising/lowering the affine indices. Likewise, we can deal with the arbitrary affine-tensor fields and their combinations ${ }^{12}$ The affine tensor $\Gamma_{a b}^{c}$ may thus be termed as the affine protoconnection.13

To account for the tensor matter fields and the continuous media it is thus sufficient to consider just the correlated pair $\theta=\vartheta \eta \vartheta^{T}$ of the affine NG fields, with transformations only under $A \in G L(4, R)$. But to include the fermion matter fields it is necessary to take into account the affine NG field $\vartheta$ itself as the basic variable, with transformations also under $\Lambda(z) \in S O(1,3)_{\text {loc }}$ to be considered below.

\subsubsection{Lorentz spinor fields}

Let $\rho(\Lambda)$ be a finite-dimensional linear representation of the Lorentz group 14 with a generic fermion field $\Psi$ transforming under $\Lambda^{\alpha \beta}=\eta^{\alpha \beta}+\lambda^{\alpha \beta}+\mathcal{O}\left(\lambda^{2}\right)$ as

$$
\Lambda(z): \Psi \rightarrow \Psi^{\prime}=\rho(\Lambda) \Psi=\left(I+\lambda^{\alpha \beta}(z) \rho\left(L_{\alpha \beta}\right)\right) \Psi+\mathcal{O}\left(\lambda^{2}\right)
$$

where $\lambda^{\alpha \beta}=-\lambda^{\beta \alpha},|\lambda| \ll 1$, are the infinitesimal (local) parameters and $L_{\alpha \beta}=-L_{\beta \alpha}$ are the generators of the Lorentz group. Now, the Lorentz anti-symmetric part $\Omega_{a}^{-\alpha \beta}$, to be called the spin-connection, allows to introduce a covariant under $S O(1,3)_{\text {loc }}$ derivative as

$$
\nabla_{a} \Psi=\partial_{a} \Psi+\Omega_{a}^{-\alpha \beta} \rho\left(L_{\alpha \beta}\right) \Psi
$$

\footnotetext{
${ }^{12}$ The property 23 is, by construction, peculiar to $\theta_{a b}$. So that an arbitrary symmetric affine tensor $T^{a b}$ formally similar to $\theta^{a b}$, generally, fulfill $\nabla_{a} T^{a b} \neq 0$.

${ }^{13}$ Moreover, $\nabla_{a}$ allows to consider in the affine space the arbitrary curvilinear coordinates, with $\Gamma_{a b}^{c}$ serving for such a purpose as a genuine affine connection.

${ }^{14}$ Note that in distinction with the affine tensors, the finite-dimensional spinors make sense only at the level of the residual Lorentz symmetry after the SSB $G L(4, R) \rightarrow S O(1,3)$. Thus the conventional matter fields may appear only after the affine SSB.
} 
where $\Omega_{a}^{-\alpha \beta}$ plays a role of the auxiliary (composed) Lorentz gauge field transforming according to eq. (14) as

$$
\Lambda(z): \Omega_{c}^{-\alpha \beta} \rightarrow \Omega_{c}^{\prime-\alpha \beta}=\Omega_{c}^{-\alpha \beta}+\lambda^{\alpha}{ }_{\gamma} \Omega_{c}^{-\gamma \beta}-\lambda^{\beta}{ }_{\gamma} \Omega_{c}^{-\gamma \alpha}-\partial_{c} \lambda^{\alpha \beta}+\mathcal{O}\left(\lambda^{2}\right) .
$$

It follows from what is shown above and the commutation relations for $L_{\alpha \beta}$ that $\nabla_{a} \Psi$ transforms under the infinitesimal $\Lambda(z)$ homogeneously like $\Psi$ itself:

$$
\Lambda(z): \nabla_{a} \Psi \rightarrow \nabla_{a}^{\prime} \Psi^{\prime} \equiv \nabla_{a}^{\prime}(\rho(\Lambda) \Psi)=\rho(\Lambda)\left(\nabla_{a} \Psi\right) .
$$

The same properties fulfill for a finite $\Lambda(z)$.

To illustrate, for a Dirac bi-spinor $\psi$ we can choose the Lorentz generators as $\rho\left(L_{\alpha \beta}\right) \sim$ $\sigma_{\alpha \beta} \equiv\left[\gamma_{\alpha}, \gamma_{\beta}\right] / 2$, with the Lorentz $\gamma$-matrices conventionally defined by the anti-commutation relations

$$
\left\{\gamma^{\alpha}, \gamma^{\beta}\right\}=2 \eta^{\alpha \beta}
$$

At that, the affine $\gamma$-matrices, $\gamma^{a} \equiv \vartheta_{\alpha}^{a} \gamma^{\alpha}$ (respectively, $\gamma_{a}=\theta_{a b} \gamma^{b}=\vartheta_{a}^{\alpha} \eta_{\alpha \beta} \gamma^{\beta}$ ), to be used in constructing the affine-invariant combinations, fulfill the relations

$$
\left\{\gamma^{a}, \gamma^{b}\right\}=2 \theta^{a b}
$$

Likewise, we can define the affine matrix $\sigma_{a b} \equiv \vartheta_{a}^{\alpha} \vartheta_{b}^{\beta} \sigma_{\alpha \beta}=\left[\gamma_{a}, \gamma_{b}\right] / 2$, etc. Such the affine matrices are to be used in constructing the Lorentz-invariant bi-linear combinations transforming only under the affine group, say, $\bar{\psi} \gamma_{a} \psi$ as the affine vector, $\bar{\psi} \gamma^{a} \nabla_{a} \psi$ as the affine scalar, $\bar{\psi} \sigma_{a b} \psi$ as the affine tensor, etc. More generally, we can consider the mixed affine-Lorentz spin-tensors $\chi_{b_{1}, \ldots}^{a_{1}, \ldots}$ to construct from them the invariant combinations both under $G L(4, R)$ and $S O(1,3)_{\text {loc. }}$.

\subsection{Second-derivative affine protogravity}

Now we are in a position to construct the affine-Goldstone NM $G / H=G L(4, R) / S O(1,3)$. First, we do this in the affine realization space and then embed the construction into the physical world manifold converting thus the latter into the pseudo-Riemannian spacetime. We restrict the subsequent consideration exclusively by the NG part of the action in terms of the protometric. The inclusion of the tensor and spinor matter fields is, in principle, straightforward by means of the techniques presented above. In the case of just the spontaneous breaking of the affine symmetry (without its explicit violation), the affine NG part of NM can depend only on $\theta^{a b}$ and its derivatives. Allowing for an explicit violation of the affine symmetry to the Lorentz one, NM may also include the Minkowski symbol $\eta_{a b}$ (or $\eta^{a b}$ ). By this token, the NG boson becomes, in fact, the pseudo-NambuGoldstone (pNG) one.

Thus, the most general Lorentz scalar Lagrangian for the affine pNG boson may generically be presented as

$$
L_{G}=L_{G}\left(\partial_{c} \theta^{a b}, \theta^{a b}, \eta_{a b}\right) .
$$

To account for $\eta_{a b}$, we may equivalently consider the Lorentz tensor

$$
H^{a}{ }_{b} \equiv \theta^{a c} \eta_{c b}
$$

(so that $\eta_{a b}=\theta_{a c} H_{b}^{c}$ ) as well as its inverse $H^{-1 b}{ }_{a} \equiv \eta^{b c} \theta_{c a}$, explicitly violating the affine symmetry to the Lorentz one. With the derivatives $\partial_{c} \theta_{a b}$ expressed through $\Gamma_{a b}^{c}$, such a 
Lagrangian may generically be partitioned into the kinetic and potential contributions as follows:

$$
L_{G}=\kappa_{0}^{2} K\left(\Gamma_{a b}^{c}, \theta^{a b}, H_{b}^{a}\right)-V\left(H_{b}^{a}\right),
$$

where the parameter $\kappa_{0}$ of the dimension of mass designates an SSB scale. The kinetic term in the second-derivative order with the minimal explicit violation of the affine symmetry is as follows:

$$
K=\frac{1}{2} \sum_{p=1}^{5} \varepsilon_{p}(H) K_{p}\left(\Gamma_{a b}^{c}, \theta_{a b}\right),
$$

where $\varepsilon_{p}, p=1, \ldots, 5$, are some free dimensionless parameters, generally, dependent on $H$, with the partial kinetic contributions

$$
\begin{array}{ll}
K_{1}=\theta^{a b} \Gamma_{a c}^{c} \Gamma_{b d}^{d}, & K_{2}=\theta_{a b} \theta^{c d} \theta^{e f} \Gamma_{c d}^{a} \Gamma_{e f}^{b}, \\
K_{3}=\theta^{a b} \Gamma_{a b}^{c} \Gamma_{c d}^{d} . & K_{4}=\theta_{a b} \theta^{c d} \theta^{e f} \Gamma_{c e}^{a} \Gamma_{d f}^{b}, \\
K_{5}=\theta^{a b} \Gamma_{a c}^{d} \Gamma_{b d}^{c}, &
\end{array}
$$

being independent of $\eta_{a b}$. Further, by admitting the second-order derivatives of $\theta$ and extending formally the affine symmetry to that under the arbitrary curvilinear coordinate transformations in the affine space ${ }^{15}$ we can formally construct from the protometric $\theta_{a b}$ a counterpart of the Riemannian tensor, then a Ricci tensor $R_{a b}$ and, at last, a Ricci scalar $R \equiv \theta^{a b} R_{a b}$. By this token, we can supplement $L_{G}$ by the term

$$
L_{g} \equiv-\frac{1}{2} \kappa_{0}^{2}\left(1+\varepsilon_{0}(H)\right) R
$$

with a dimensionless parameter $\varepsilon_{0}$. And finally, the potential term $V(H)$ is an arbitrary Lorentz-scalar polynomial of $H^{a}{ }_{b}$, like $\operatorname{tr}\left(H^{2}\right) \equiv H^{a}{ }_{b} H^{b}{ }_{a}$, etc., supplemented, generally, by $\operatorname{det}\left(H_{b}^{a}\right)=\operatorname{det}\left(\theta^{a b}\right) \operatorname{det}\left(\eta_{a b}\right)$, as well as a constant part $V_{0}$.

Likewise, we could construct in the affine realization space, a progenitor of the Lorentz spacetime, the most general affine and locally-Lorentz invariant NM for the pNG boson supplemented by the matter fields. Nevertheless, such a construction is to be considered as no more than an auxiliary one. To describe the real world, the construction should be proliferated onto an arbitrary world manifold, a progenitor of the pseudo-Riemannian spacetime, to be considered below.

\section{Quartet-metric EFT and beyond}

\subsection{General covariance}

Let now the real world - a set of the primary events (points) - be modeled by a fourdimensional topological manifold $M^{4}$ endowed with the arbitrary smooth enough coordinates $x^{\mu}, \mu=0, \ldots, 3$. (As before, the index 0 is originally nothing but a notation.) Let $M^{4} \leftrightarrow R^{4}$ be a local one-to-one mapping of the manifold onto the affine realization space by means of some invertible transformation functions $z^{a}=Z^{a}(x)$, with $x^{\mu}=x^{\mu}(z)$. Call

\footnotetext{
${ }^{15}$ To generate such transformations in the affine space it is, in fact, sufficient to consider a closure of the affine and conformal transformations 12 .

${ }^{16}$ In principle, $R$ may be expressed, up to a surface contribution, through a linear combination of $K_{3}$ and $K_{5}$ (or rather, say, $K_{3}$ may be expressed through $K_{5}$ and $R$, etc,). Not having a prior preference, we leave explicitly all the terms shown above.
} 
the so-constructed distinct coordinates $z^{a}=Z^{a}(x)$ by definition the quasi-affine ones on $M^{4}$. Generally, the reversibility of the mapping may be hampered by some singular subsets of $M^{4}$, with the mapping being in fact patch-wise. In terms of $z^{a}$, let us map all the quantities on $R^{4}$ into the respective quantities on $M^{4}$. A priori, the mapping given by $Z^{a}(x)$ could remain unspecified signifying ultimately an incompleteness of the approach. To eliminate this uncertainty we treat $Z^{a}(x)$ by construction as the dynamical variables describing gravity on par with $\theta_{a b}$. Otherwise, this means that the gravity is a net result of imposing both the local field-theoretic (due to $\theta_{a b}$ ) and geometrical (due to $Z^{a}$ ) effects. For the general covariance (GC) let us express further the so-obtained structures in terms of the arbitrary observer's coordinates $x^{\mu}=x^{\mu}(z)$. Introducing for such the transformations on $M^{4}$ the respective quasi-affine tetrad $\zeta_{\mu}^{a}(x) \equiv \partial_{\mu} Z^{a}$ and its inverse, $\zeta_{a}^{\mu}(x) \equiv \partial x^{\mu} /\left.\partial z^{a}\right|_{z=Z(x)}$, we can proliferate NM from the affine realization space in the GC manner onto the world manifold converting the latter into the pseudo-Riemannian one. Operationally, this can be achieved from the preceding results through the coordinate substitution $z^{a} \rightarrow x^{\mu}$ followed by the proper substitutions of the basic quantities, which may be partitioned in the three groups as shown below.

\subsubsection{Enhanced contributions}

The first group of substitutions is as follows:

$$
\begin{aligned}
\theta_{a b} & \rightarrow g_{\mu \nu} \equiv \zeta_{\mu}^{a} \zeta_{\nu}^{b} \theta_{a b}, \\
\theta^{a b} & \rightarrow g^{\mu \nu} \equiv \zeta_{a}^{\mu} \zeta_{b}^{\nu} \theta^{a b}, \\
R_{a b} & \rightarrow R_{\mu \nu} \equiv \zeta_{\mu}^{a} \zeta_{\nu}^{b} R_{a b}, \\
R \equiv \theta^{a b} R_{a b} & \rightarrow R \equiv g^{\mu \nu} R_{\mu \nu}, \\
\operatorname{det}\left(\theta_{a b}\right) & \rightarrow g \equiv \operatorname{det}\left(g_{\mu \nu}\right)=\operatorname{det}\left(\zeta_{\mu}^{a}\right)^{2} \operatorname{det}\left(\theta_{a b}\right), \\
\gamma^{a} & \rightarrow \gamma^{\mu} \equiv \zeta_{a}^{\mu} \gamma^{a},
\end{aligned}
$$

with

$$
\left\{\gamma^{\mu}, \gamma^{\nu}\right\}=2 g^{\mu \nu}
$$

In what is shown above, the NG field in disguise, $g_{\mu \nu}$, is to be treated as the pseudoRiemannian metric with the inverse $g^{\mu \nu}$, while $R_{\mu \nu}$ proves to be the conventional Ricci curvature tensor constructed from $g_{\mu \nu}$. This group of term is relevant for GR and its direct siblings.

Otherwise, the quantities above may be expressed through a local-Lorentz tetrad $\vartheta_{\mu}^{\alpha}$ defined as

$$
\vartheta_{a}^{\alpha} \rightarrow \vartheta_{\mu}^{\alpha} \equiv \zeta_{\mu}^{a} \vartheta_{a}^{\alpha}
$$

so that, say,

$$
\begin{aligned}
g_{\mu \nu} & =\vartheta_{\mu}^{\alpha} \vartheta_{\nu}^{\beta} \eta_{\alpha \beta}, \\
\sqrt{-g} & =\operatorname{det}\left(\vartheta_{\mu}^{\alpha}\right)\left(-\operatorname{det}\left(\eta_{a b}\right)\right)^{1 / 2} \\
\gamma^{\mu} & =\vartheta_{\alpha}^{\mu} \gamma^{\alpha},
\end{aligned}
$$

etc. In principle, the local-Lorentz tetrad $\vartheta_{\mu}^{\alpha}$ may be chosen as an alternative to the metric $g_{\mu \nu}$. At that, the tetrad $\vartheta_{\mu}^{\alpha}(X)$ in an arbitrary chosen point $X$ may be associated with the so-called locally-inertial tetrad $\hat{\vartheta}_{\mu}^{\hat{\alpha}}(X)$ in GR through a locally-inertial gauge given by $\hat{\Lambda}(X)$ as

$$
\left.\hat{\Lambda}_{\beta}^{\alpha}(X) \vartheta_{\mu}^{\beta}(X)\right|_{\alpha=\hat{\alpha}}=\hat{\vartheta}_{\mu}^{\hat{\alpha}}(X) \equiv \partial \hat{x}_{X}^{\hat{\alpha}} /\left.\partial x^{\mu}\right|_{x=X},
$$


were $\hat{x}_{X}^{\hat{\alpha}}$ are the locally-inertial in a vicinity of the point $X$ coordinates ${ }^{17}$ Due to the local-Lorentz symmetry using the locally-inertial coordinates to describe fermions is not as crucial as in the plain GR. For GR and its direct siblings, the dependence on $Z^{a}$ gets completely hidden, with such the theories becoming the exclusively metric ones ${ }^{18}{ }^{19}$

The quasi-affine coordinates manifests themselves only for the GR extensions originating from terms in the affine realization space with the normal or suppressed strength. The proper terms correspond to the non-enhanced affine symmetry, respectively, without or with an explicit violation of the affine symmetry to the Lorentz one, presented below.

\subsubsection{Normal contributions}

The second group of terms consists of the connection-like GC tensor

$$
\Gamma_{a b}^{c} \rightarrow B_{\mu \nu}^{\lambda} \equiv \Gamma_{\mu \nu}^{\lambda}-\gamma_{\mu \nu}^{\lambda}
$$

where

$$
\Gamma_{\mu \nu}^{\lambda} \equiv \zeta_{c}^{\lambda} \zeta_{\mu}^{a} \zeta_{\nu}^{b} \Gamma_{a b}^{c}=\frac{1}{2} g^{\lambda \kappa}\left(\partial_{\mu} g_{\nu \kappa}+\partial_{\nu} g_{\mu \kappa}-\partial_{\kappa} g_{\mu \nu}\right)
$$

is nothing but the Christoffel connection for the pseudo-Riemannian metric $g_{\mu \nu}$ and

$$
\gamma_{\mu \nu}^{\lambda} \equiv \zeta_{a}^{\lambda} \partial_{\mu} \zeta_{\nu}^{a}=\zeta_{a}^{\lambda} \partial_{\nu} \zeta_{\mu}^{a}
$$

is the inhomogeneously transforming part of the connection under a change of the coordinates. The contribution of $B_{\mu \nu}^{\lambda}$ signifies the "hard"/kinetic extension to GR and clearly requires both the local-Lorentz and quasi-affine tetrads, respectively, $\vartheta_{\mu}^{\alpha}$ and $\zeta_{\mu}^{a}$.

\subsubsection{Suppressed contributions}

At last, the third group of terms is as follows:

$$
\begin{aligned}
\eta_{a b} & \rightarrow \zeta_{\mu \nu} \equiv \zeta_{\mu}^{a} \zeta_{\nu}^{b} \eta_{a b} \\
\eta^{a b} & \rightarrow \zeta^{\mu \nu} \equiv \zeta_{a}^{\mu} \zeta_{b}^{\nu} \eta^{a b} \\
\operatorname{det}\left(\eta_{a b}\right) & \rightarrow \zeta \equiv \operatorname{det}\left(\zeta_{\mu \nu}\right)=\operatorname{det}\left(\zeta_{\mu}^{a}\right)^{2} \operatorname{det}\left(\eta_{a b}\right) \\
H_{b}^{a} \equiv \theta^{a c} \eta_{c b} & \rightarrow H^{\mu}{ }_{\nu}=\zeta_{a}^{\mu} \zeta_{\nu}^{b} H_{b}^{a}=g^{\mu \lambda} \zeta_{\lambda \nu} .
\end{aligned}
$$

Call $\zeta_{\mu \nu}$ the quasi-Minkowskian metric. The terms above originate clearly from those in the affine realization space containing $\eta_{a b}$ and imply thus an explicit affine symmetry violation ${ }^{20}$ Clearly, the tetrad $\zeta_{\mu}^{a} \equiv \partial_{\mu} Z^{a}$ defines the (patch-wise) quasi-affine coordinates $z^{a}=Z^{a}(x)$, wherein $\zeta_{a b}(z)=\eta_{a b}$ and $\gamma_{a b}^{c}(z)=0$. At that, the term $\gamma_{\mu \nu}^{\lambda}$ eq. 433 defined originally through $\zeta_{\mu}^{a}$ (and its inverse) may equivalently be presented as the Christoffel connection corresponding to the quasi-Minkowskian metric $\zeta_{\mu \nu}$, with $B_{\mu \nu}^{\lambda}$ getting explicitly the GC tensor. Nevertheless, the dependence on $\eta_{a b}$, in fact, drops off and the term

\footnotetext{
${ }^{17}$ Remind that such coordinates are defined as those wherein the metric is locally-flat: $\left.\hat{g}_{\hat{\alpha} \hat{\beta}}\left(\hat{x}_{X}\right)\right|_{\hat{x}_{X} \simeq X} \simeq$ $\eta_{\hat{\alpha} \hat{\beta}}$ up to the quadratic deviations of $\hat{x}_{X}$ from $X$.

${ }^{18}$ Hence, restricting $a b$ initio NM by the pseudo-Riemannian manifold $M^{4}$ [9] we could in the end recover only the part of NM corresponding to GR (and its direct siblings). For this reason, the two-stage realization of NM to get the quartet-modified GR is paramount.

${ }^{19}$ The respective group of terms originates ultimately from those in the affine realization space with the affine symmetry enhanced to the conformal one, as it was mentioned previously.

${ }^{20}$ In particular, this concerns the quasi-Minkowskian measure $\sqrt{-\zeta}$.
} 
$B_{\mu \nu}^{\lambda}$ does not violates the affine symmetry. ${ }^{21}$ Finally remark that were $z^{a}=Z^{a}(x)$ not the dynamical but some prior/"absolute" coordinates the affine-Goldstone NM would be either incomplete (under retaining only the first group of terms shown above, which are expressed entirely through the local-Lorentz tetrad $\zeta_{\mu}^{\alpha}$ absorbing $Z^{a}$ ) or unclosed (under addition of the two last groups of terms dependent also on the quasi-affine tetrad $\left.\zeta_{\mu}^{a}\right)$.

\subsection{Second-derivative reduction}

The gravity action $I$ in a world manifold $M^{4}$ looks generically like

$$
I=\int_{M^{4}} L_{G} \mathcal{M} d^{4} x
$$

with $L_{G}$ a GC scalar Lagrangian and $\mathcal{M}$ a spacetime measure, i.e., a GC scalar density of the proper weight for $I$ to be a GC scalar. At that, due to a prior freedom of redefining the Lagrangian there is an arbitrariness of choosing the measure. E.g., the latter may be defined as $\sqrt{-g}$ or $\sqrt{-\zeta}$, or as a combination of both. By this token, the action for the pure gravity may be expressed without loss of generality through the metric $g_{\mu \nu}$ and the field $H^{\mu}{ }_{\nu}$ as follows:

$$
I=\int L_{G}\left(\partial_{\lambda} g_{\mu \nu}, g_{\mu \nu}, H^{\mu}{ }_{\nu}\right) \sqrt{-g} d^{4} x .
$$

The second-derivative Lagrangian in the GC form now becomes

$$
L_{G}=L_{g}+\kappa_{\mathrm{P}}^{2} K\left(B_{\mu \nu}^{\lambda}, g_{\mu \nu}, H^{\mu}{ }_{\nu}\right)-V\left(H^{\mu}{ }_{\nu}\right),
$$

with

$$
L_{g}=-\frac{1}{2} \kappa_{\mathrm{P}}^{2}\left(1+\varepsilon_{0}(H)\right) R,
$$

where $R$ is the conventional Ricci scalar constructed from the Christoffel connection $\Gamma_{\mu \nu}^{\lambda}\left(g_{\mu \nu}\right){ }^{22}$ Here we have identified the affine SSB scale $\kappa_{0}$ with the reduced Planck mass $\kappa_{\mathrm{P}}=1 / \sqrt{8 \pi G_{\mathrm{N}}}$, where $G_{\mathrm{N}}$ is the Newton's constant.

The kinetic term $K$ looks as before

$$
K=\frac{1}{2} \sum_{p} \varepsilon_{p}(H) K_{p}\left(B_{\mu \nu}^{\lambda}, g_{\mu \nu}\right)
$$

with the partial contributions as follows:

$$
\begin{array}{ll}
K_{1}=g^{\mu \nu} B_{\mu \kappa}^{\kappa} B_{\nu \lambda}^{\lambda}, & K_{2}=g_{\mu \nu} g^{\kappa \lambda} g^{\rho \sigma} B_{\kappa \lambda}^{\mu} B_{\rho \sigma}^{\nu}, \\
K_{3}=g^{\mu \nu} B_{\mu \nu}^{\kappa} B_{\kappa \lambda}^{\lambda}, & K_{4}=g_{\mu \nu} g^{\kappa \lambda} g^{\rho \sigma} B_{\kappa \rho}^{\mu} B_{\lambda \sigma}^{\nu}, \\
K_{5}=g^{\mu \nu} B_{\mu \kappa}^{\lambda} B_{\nu \lambda}^{\kappa} . &
\end{array}
$$

The potential $V(H)$ remains as before, with $H_{b}^{a}$ substituted by $H^{\mu}{ }_{\nu}=g^{\mu \lambda} \zeta_{\lambda \nu}$. The simplest case is given under dependence of $K$ only on a scalar-graviton field

$$
\sigma \equiv \ln \left(\operatorname{det}\left(H^{\mu}{ }_{\nu}\right)\right)^{-1 / 2}=\ln \sqrt{-g} / \sqrt{-\zeta}
$$

\footnotetext{
${ }^{21} \mathrm{~A}$ small explicit violation of the affine symmetry through $\eta_{a b}$ may, in principle, serve as a raison d'être inducing the affine SSB, the latter surviving even after such a "seed" violation dropping-off.

${ }^{22}$ By default, the indices of $H^{\mu}{ }_{\nu}=g^{\mu \lambda} \zeta_{\lambda \nu}, H^{-1 \mu}{ }_{\nu}=\zeta^{\mu \lambda} g_{\lambda \nu}$, etc., are raised or lowered, if necessary, through $g^{\mu \nu}$ or $g_{\mu \nu}$, respectively.
} 
with $\varepsilon_{1} \neq 0$ and the rest of $\varepsilon_{p}$ 's being zero, so that

$$
K=\frac{1}{2} \varepsilon_{1}(\sigma) K_{1}=\frac{1}{2} \varepsilon_{1}(\sigma) g^{\mu \nu} \partial_{\mu} \sigma \partial_{\nu} \sigma
$$

supplemented by a potential $V=V(\sigma)$. Likewise, we could reproduce in the affineGoldstone NM the more elaborate cases for the quartet-metric gravity [2, 3].

\subsection{Weak-field limit}

Let now the dynamical gravity fields $Z^{a}$ and $g_{\mu \nu}$ are decomposed as

$$
\begin{aligned}
Z^{a} & =\bar{Z}^{a}+\zeta^{a}, \\
g_{\mu \nu} & =\bar{g}_{\mu \nu}+h_{\mu \nu},
\end{aligned}
$$

with the backgrounds $\bar{Z}^{a}$ and $\bar{g}_{\mu \nu}$ supplemented by the small dynamical deviations $\zeta^{a}$ and $h_{\mu \nu}$, respectively. Choosing the quasi-affine coordinates $z^{a}=\bar{Z}^{a}(x)$, so that $\bar{\zeta}_{a b}=\eta_{a b}$, and assuming for simplicity $\bar{g}_{a b}=\eta_{a b}$ we get (operating indices now through $\eta_{a b}$ and $\eta^{a b}$ ) in the linear approximation (LA) as follows:

$$
\begin{aligned}
\zeta_{a b} & =\eta_{a b}+\partial_{a} \zeta_{b}+\partial_{b} \zeta_{a} \\
H_{a b} & =\eta_{a b}-h_{a b}+\partial_{a} \zeta_{b}+\partial_{b} \zeta_{a} .
\end{aligned}
$$

Likewise, the kinetic term proves to exhibit the similar substitution:

$$
h_{a b} \rightarrow h_{a b}^{\prime}=h_{a b}-\left(\partial_{a} \zeta_{b}+\partial_{b} \zeta_{a}\right) .
$$

This means that there takes place the Higgs-like SSB of the quartet-metric gravity, with converting the originally gauge components of the metric into the physical ones to be treated as the dark substances of the Universe, in addition to the tensor graviton. Just to illustrate a variety of the arising possibilities we consider in what follows the two extreme cases under choosing for simplicity the flat backgrounds: $\bar{g}_{a b}=\bar{\zeta}_{a b}=\bar{H}_{a b}=\eta_{a b}$.

\subsubsection{Pure-tensor gravity}

In one extreme case, imposing on $\bar{\varepsilon}_{p} \equiv \varepsilon_{p}(\bar{H})$ the constraints

$$
\begin{aligned}
\bar{\varepsilon}_{1}=0, & \bar{\varepsilon}_{2}=-\bar{\lambda}, \\
\bar{\varepsilon}_{3}=\bar{\varepsilon}_{t}-\bar{\lambda}, & \bar{\varepsilon}_{4}=\bar{\lambda}, \\
\bar{\varepsilon}_{5}=-\bar{\varepsilon}_{t}+3 \bar{\lambda}, &
\end{aligned}
$$

with $\bar{\varepsilon}_{t}$ and $\bar{\lambda}$ some free parameters, we recover for $h_{a b}^{\prime}$ in LA the conventional GR Lagrangian in an obvious notation as follows:

$$
L_{G}=\frac{1}{8} \kappa_{\mathrm{P}}^{2}\left(1+\bar{\varepsilon}_{0}+\bar{\varepsilon}_{t}\right)\left(\left(\partial_{c} h^{\prime a b}\right)^{2}-2\left(\partial_{a} h^{\prime a b}\right)^{2}+2 \partial_{a} h^{\prime a b} \partial_{b} h_{c}^{\prime c}-\left(\partial_{a} h_{b}^{\prime b}\right)^{2}\right),
$$

without modification of the tensor gravity. At that, $\bar{\lambda}$ drops off completely, with $\bar{\lambda}=0$ in the simplest version. To reproduce GR in LA exactly we should additionally put $\bar{\varepsilon}_{g} \equiv \bar{\varepsilon}_{0}+\bar{\varepsilon}_{t}=0$, recovering the Newton's gravity in LA precisely. The weak-field post-Newtonian contributions to $L_{G}$ impose, generally, some restrictions on the leftout parameters $\bar{\varepsilon}_{t}$ and $\bar{\lambda}$. The deviations from the relations shown above would imply 
some additional kinetic contributions beyond GR already in LA, being, as it could be anticipated strongly suppressed. At last, the potential $V_{t}\left(h^{\prime a b}\right)$ may be chosen so to recover in LA the Fiertz-Pauli term for the massive tensor graviton possessing, due to the Higgs-like mechanism, the descent massless limit. Nevertheless, even at the zero deviations from GR in LA the full nonlinear theory may still essentially deviate from GR in the strong-field limit producing some additional restrictions/predictions.

\subsubsection{Scalar-tensor gravity}

In other extreme case, with $\bar{\varepsilon}_{0}=0$ and

$$
\begin{array}{cl}
\bar{\varepsilon}_{1}=\bar{\varepsilon}_{s}, & \bar{\varepsilon}_{2}=-\bar{\lambda}, \\
\bar{\varepsilon}_{3}=-\bar{\lambda}, & \bar{\varepsilon}_{4}=\bar{\lambda}, \\
\bar{\varepsilon}_{5}=3 \bar{\lambda}, &
\end{array}
$$

where $\bar{\varepsilon}_{s}$ is a free parameter corresponding to the addition of the scalar graviton $\sigma=$ $h_{c}^{\prime c} / 2 \equiv h^{\prime} / 2$ through

$$
K=\frac{1}{8} \bar{\varepsilon}_{s} \partial_{a} h^{\prime} \partial^{a} h^{\prime}
$$

without modifying tensor gravity. With $\bar{\lambda}$ dropped=off completely, one can put $\bar{\lambda}=0$ in the simplest version. This kinetic term should be supplemented by a potential $V_{s}\left(h^{\prime}\right)$ for the scalar-graviton mass and self-interactions. A priori, there is also possible to consider a (putatively problematic) vector graviton, as well as a mixture of the different kinds of gravitons. Such the additional gravity components, having the NG origin common with the tensor graviton, may naturally be treated as the gravitational dark substances (DM, DE, etc.) of the Universe.

\section{Summary: NM vs. EFT and beyond}

Let us briefly summarize the main advantages provided for the quartet-modified GR by the NM vs. EFT frameworks:

- NM justifies the extended set of the gravity fields, $g_{\mu \nu}$ and $Z^{a}$, as well as the pattern of the basic symmetries: GC and the global Poincare symmetry, the latter introduced in EFT ad hock.

- NM refines the types of the gravity interactions and their hierarchy as originating from the enhanced, normal and suppressed terms in the affine realization space.

- NM ascribes the clear-cut physical meaning to the Planck scale $\kappa_{\mathrm{P}}$, determining the strength of the gravity interactions, as a scale of the affine SSB.

- NM states the unique tensor graviton and the putative gravitational dark substances (DM, DE, etc.) as the emergent pNG bosons corresponding to the affine SSB.

- NM may ultimately serve as a link between the quartet-metric EFT of gravity below the Planck scale and the producing NM underlying theory above this scale, pointing, arguably, towards the gravity and spacetime as emerging at $\kappa_{\mathrm{P}}$ due to the affine SSB. 
Altogether, in the paper there is proposed the route of the GR modification, so to say, "in width" and "in depth" as follows:

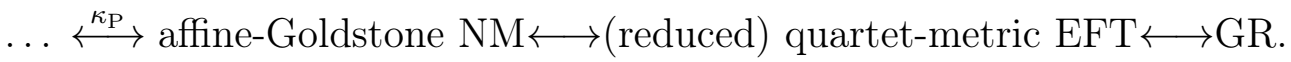

For studying the manifestations of gravity it is sufficient to stay at the level of EFT, but for a deeper revealing the nature of gravity it is necessary to adhere to the more profound level given by NM. The respective two-level phenomenon, being the affineGoldstone in its nature and the quartet-metric in its appearance, may be called the affine-Goldstone/quartet-metric gravity. Further studying the latter, both theoretically and phenomenologically, to verify its validity is urgent.

Acknowledgments The author is grateful to S. S. Gershtein for interest to the work and encouragement, and to V. V. Kabachenko for discussions.

\section{Appendix: SSB generalities}

Cosets To describe group-theoretically the SSB $G \rightarrow H$ of a global continuous internal symmetry/group $G$ to its subgroup $H \subset G$ we should first clarify the notion of the socalled cosets. A (say, left) coset of a subgroup $H$ in the group $G$ with respect to an element $k \in G$ is defined generically as a subset (not, generally, a subgroup) of $G$ equivalent to the given $k$ modulo the (right) multiplication by any $h \in H$, i.e., $k \sim k h$. The cosets are either identical or disjoint. Each element of $G$ belongs to one and only one coset, with the cosets partitioning the group, i.e., the unification of all the cosets represents the whole group. At that, the coset as a whole is uniquely determined by any its element chosen as a representative, say, $k$. The total set of the (left) coset representatives, $K=\{k\}$, constitutes the (left) coset space $K=G / H$. The coset space ultimately serves as a space for NRs and NMs to implement SSB, with the cosets providing a "language" for SSB.

SSB and (pseudo-)Nambu-Goldstone bosons Now, if $G$ is a symmetry group of a physical system, let $\mid I>$ be the system ground state ("vacuum") invariant under $G$, i.e., $g|I>=| I>$ for any $g \in G$. Let now there takes place SSB $G \rightarrow H$ meaning that the invariance of the vacuum lowers up to $H \subset G$, i.e., only $h|I>=| I>$ for any $h \in H$. In this case, for an arbitrary $g \in G$, with the decomposition $g=k h, k \in K$ and $h \in H$, there takes place $g|I>=k| I>\equiv|k>\neq| I>$. This corresponds to SSB in the NG mode, with the appearance of a set of the degenerate vacua $\mid k>, k \in K$, the excitation of which corresponds to the physical NG bosons. Under an explicit violation of $G$, the NG bosons become, in fact, the pseudo-NG (pNG) ones. In these terms, the field variable on a flat spacetime describing the SSB $G \rightarrow H$ in the NG mode may conventionally be chosen as the coset (local) representative $k(x) \in K$ for $x \in R^{4}$.

Nonlinear realizations Further, the result of the action of a group element $g \in G$ on a (left) coset representative $k \in K, g k$, being a group element may as well be decomposed as $g k=k^{\prime} h$, with a new $k^{\prime} \in K$ and some $h=h(g, k) \in H$. This implies that the coset representative $k$ transforms under $G$ nonlinearly as

$$
g: k \rightarrow k^{\prime}=g k h^{-1}(g, k) .
$$


For $g=h_{0} \in H$, there can be shown that $h\left(h_{0}, k\right)=h_{0}$ and, in particular, $h(I, k)=I$, with $k^{\prime}=k$. This defines NR of the group $G$ on the (left) coset space $K=G / H$ for the SSB $G \rightarrow H$.

Nonlinear models And finally, a NM describing the NG field $k \in K=G / H$ appearing due to SSB $G \rightarrow H$ for a pair of the global internal groups $H \subset G$ is a specific field theory for the coset (local) representatives $k(x) \in K, x \in R^{4}$. Such a theory may be defined by an action $S=\int L(k, \psi) d^{4} x$, with a Lagrangian $L$ invariant under the nonlinearly realized/"hidden" symmetry $G$. At that, a generic matter field $\psi$ transforms as a linear representation $\rho$ of the residual (classification) subgroup $H \subset G$ through $h(g, k)$ determined above:

$$
g: \psi \rightarrow \psi^{\prime}=\rho(h(g, k)) \psi,
$$

with the NG field $k$ being thus omnipresent (what, by the way, is characteristic of graviton). Under $g=h_{0} \in H$, this becomes the conventional linear representation $\rho\left(h_{0}\right)$ of $H \subset G$. This generically defines NM for the SSB $G \rightarrow H$ built on NR of the group $G$, reducing to the linear representation when restricted by the unbroken subgroup $H \subset G$.

The affine-Goldstone NM for gravity considered in the given paper corresponds (with a modification) to the symmetry pattern $G / H=G L(4, R) / S O(1,3)$. As the pNG bosons there serve here the (in a general case, massive) tensor graviton and the additional gravity components, to be associated ultimately with the gravitational DM and DE.

\section{References}

[1] C. Patrignani et. al., Review of Particle Physics, Cin. Phys. C 40, 100001 (2016).

[2] Y. F. Pirogov, Quartet-metric general relativity: scalar graviton, dark matter and dark energy, Eur. Phys. J. C 76, 215 (2016); arXiv:1511.04742 [ge-gc].

[3] Y. F. Pirogov, Quartet-metric gravity and dark components of the Universe, Proc. XXXI Int. Workshop on HEP: Critical Points in the Modern Particle Physics, Protvino, Russia, 5-7 July 2017, Int. J. Mod. Phys.: Conf. Series, 47, 1860101 (2018); arXiv:1712.00612 [gr-gc].

[4] C. Isham, A. Salam, J. Strathdee, Nonlinear realizations of spacetime symmetries. Scalar and tensor gravity, Ann. Phys. (NY) 62, 98 (1971).

[5] A. B. Borisov, V. I. Ogievetsky, Theory of dynamical affine and conformal symmetries as the theory of the gravitational field, Teor. Mat. Fiz. 21, 329 (in Russian) (1974); Preprint JINR E-7684 (in English) (JINR, 1974).

[6] S. R. Coleman, J. Wess, B. Zumino, Structure of phenomenological Lagrangians. I, Phys. Rev. D 177, 2239 (1969).

[7] C. G. Callan, S. R. Coleman, J. Wess, B. Zumino, Structure of phenomenological Lagrangians. II, Phys. Rev. D 177, 2247 (1969).

[8] A. Salam, J. Strathdee, Nonlinear realizations. I. The role of Goldstone bosons, Phys. Rev. D 184, 1750 (1969).

[9] C. J. Isham, Metric structures and chiral symmetries, Nuovo Cim. A 61, 188 (1969). 
[10] Y. F. Pirogov, Gravity as the affine Goldstone phenomenon and beyond, Yad. Fiz. 68, 1966 (2005) [Phys. Atom. Nucl. 68, 1904 (2005)]; arXiv:gr-qc/0405110.

[11] G. Sardanashvily, Gravity as a Goldstone field in the Lorentz gauge theory, Phys. Lett. A 75, 257 (1980).

[12] V. I. Ogievetsky, Infinite-dimensional algebra of general covariance group as the closure of finite-dimensional algebras of conformal and linear groups, Lett. Nuovo Cim. 8, 988 (1973). 